\title{
AMOSTRAGEM DE DISCOS E USO DE EQUAÇÕES PARA ESTIMAR A DENSIDADE BÁSI- CA DA MADEIRA EM DIVERSAS FITOFISIONOMIAS
}

\author{
DISC SAMPLING AND USE OF EQUATIONS FOR ESTIMATE THE BASIC WOOD DENSITY IN \\ SEVERAL PHYTOPHYSIOGNOMIES
}

\begin{abstract}
Gabriel Marcos Vieira Oliveira ${ }^{1}$ José Márcio de Mello $^{2}$ João Domingos Scalon ${ }^{3}$ José Roberto Soares Scolforo $^{2}$ Thiago Campos Monteiro ${ }^{4}$
\end{abstract}

\section{RESUMO}

O objetivo deste trabalho foi determinar o número adequado de discos de madeira, as melhores posições de amostragem, ao longo do fuste e propor equações que permitam estimar a densidade básica da madeira de árvores, a partir de um número reduzido de discos, em diversas fisionomias florestais, para subsidiar estimativas de biomassa. Os dados de cubagem rigorosa das árvores foram obtidos no Inventário Florestal de Minas Gerais, advindas de 1.325 árvores, distribuídas em regiões, classes de diâmetro e altura e em diversas fisionomias florestais. De cada árvore foram retirados cinco discos de madeira nas posições 0 (A), 25 (B), 50 (C), 75 (D) e 100\% (E) da altura comercial. A densidade básica da madeira de cada disco foi determinada, conforme a ABNT NBR 11.941 (2003), e a densidade geral do fuste, a partir da média aritmética entre as densidades dos discos. Os dados foram analisados, com base na análise de regressão, critério de informação de Akaike, erro padrão da estimativa e análise gráfica de resíduos. Os resultados indicaram que, em geral, a melhor posição de amostragem, para estimativa da densidade básica da madeira do fuste com apenas um disco, deve ser efetuada na metade do fuste principal, com dois discos nas posições 25 e $75 \%$ da altura e com três discos nas posições 0,50 e $100 \%$ da altura do fuste. Observou-se, também, que a melhor estimativa da densidade básica do fuste sempre será dada por aquela com maior número de discos, entretanto, é possível estimar com alta precisão a densidade do fuste com menor número de discos, desde que amostrados em posições específicas do fuste e inseridos nas equações propostas de acordo com cada fitofisionomia. Palavras-chave: modelagem; posição longitudinal; folhosas; inventário florestal.

\begin{abstract}
The objective of this study was to determine the most suitable number of discs, the best sampling positions along the stem and to propose equations to estimate the basic density of trees using a reduced number of discs in various phytophysiognomies, in order to subsidize biomass estimates. The detailed scaling data of the trees were obtained from the Forest Inventory of Minas Gerais state, and is composed by 1,325 trees distributed in regions, diameters and height classes and various forest formations. We took five discs from each at positions 0 (A), 25 (B), 50 (C) 75 (D) and 100\% (E) of commercial height. The density of each disk was determined according to ABNT NBR 11.941 (2003) and the overall stem density from the arithmetic mean of the disc densities. Analyses were performed by regression analysis, the Akaike Information Criterion, Standard Error of the Estimate and graphical analysis of residuals. The results indicated that, in

1 Engenheiro Florestal, Dr., Agente em Desenvolvimento Agropecuário, IDAF, Rua Dr. José Coelho dos Santos, 231, CEP 29400-000, Mimoso do Sul (ES), Brasil. gabrielmvo@gmail.com

2 Engenheiro Florestal, Dr., Professor do Departamento de Ciências Florestais, Universidade Federal de Lavras, Laboratório de Estudos e Projetos em Manejo Florestal, Campus Universitário, CEP 37200-000, Lavras (MG), Brasil.josemarcio@dcf.ufla.br/jscolforo@dcf.ufla.br

3 Estatístico, Dr., Professor do Departamento de Estatística, Universidade Federal de Lavras, Campus Universitário, CEP 37200-000, Lavras (MG), Brasil. scalon@des.ufla.br

4 Engenheiro Florestal, Dr., Professor do Departamento de Engenharia e Tecnologia Florestal, Universidade Federal do Paraná, CIFLOMA, Av. Pref. Lothário Meissner, 632, Jardim Botânico, CEP: 80210-170, Curitiba (PR), Brasil. thiago.monteiro@ufpr.br
\end{abstract}

Recebido para publicação em 8/01/2014 e aceito em 4/09/2017

Ci. Fl., v. 28, n. 4, out. - dez., 2018 
general, the best sampling position for estimating the basic density of stem wood with only one disc should be performed in the middle of the main stem, with two discs in positions 25 and $75 \%$ in height and with three discs at positions 0,50 and $100 \%$ of the stem height. We also observed that the best estimate of stem density will always be given by the largest number of discs. However, it is possible to estimate the density of the trees with high precision, with a smaller number of discs provided that they are sampled at specific positions of the stem and inserted in the Equations according to each phytophysiology.

Keywords: modeling; longitudinal position; hardwood; forest inventory.

\section{INTRODUÇÃO}

A biomassa ou peso seco podem ser considerados uma medida da produção e da produtividade florestal. A quantificação destaca-se para fins energéticos, e do ponto de vista mais contemporâneo, essa variável pode ser convertida em massa de carbono que, por sua vez, subsidia estimativas de emissão e sequestro de gases do efeito estufa na atmosfera (NOGUEIRA et al., 2007; FEARNSIDE, 2008; SILVEIRA, 2010).

As determinações de biomassa convencionais referem-se, principalmente, à biomassa do estrato arbóreo da árvore acima do solo o qual é de mais fácil mensuração e contribui significativamente com a biomassa total no sistema florestal, visto que, dentro dele, o tronco ou fuste compõe a fração mais representativa da biomassa na árvore e da biomassa total da floresta, por isso, sua determinação deve ser feita da forma mais precisa possível (SANQUETTA, 2002; VIEIRA; SANQUETTA; BARBEIRO, 2009).

A determinação da biomassa do fuste é normalmente realizada por meio da técnica volumétrica pela qual a estimativa do peso seco é obtida com o produto do volume da árvore e com a densidade básica da sua madeira. O volume da árvore pode ser determinado por algum método de cubagem rigorosa, e a densidade básica por discos de madeira devidamente amostrados, ao longo do fuste e, na maioria das vezes, são amostrados cinco discos (SCOLFORO et al., 2004; SILVEIRA et al., 2008; VISMARA, 2009).

No entanto, a cubagem rigorosa é uma atividade que exige técnica, tempo e recursos para ser executada, assim como a retirada dos discos e a determinação da densidade básica da madeira em laboratório. Uma alternativa, para se reduzir esse esforço, é aprimorar a amostragem dos discos para determinação da densidade básica da madeira do fuste, de forma a retirá-los das posições mais representativas e, consequentemente, reduzir o número de discos amostrados. Essa alternativa pode garantir ganho de tempo, recursos e, também, garantir a manutenção da precisão das estimativas de biomassa, uma vez que, segundo Woodcock (2000), a densidade da madeira é um dos fatores-chave nos cálculos a serem efetuados.

Desse modo, o objetivo deste trabalho foi determinar o número mais adequado de discos de madeira, as melhores posições de amostragem, ao longo do fuste e propor equações que permitam estimar a densidade básica da madeira do fuste de árvores, utilizando um número reduzido de discos, em diversas fisionomias florestais, para subsidiar estimativas de biomassa florestal.

\section{MATERIAL E MÉTODOS}

\section{Origem dos dados}

Os dados utilizados neste trabalho foram obtidos do Inventário Florestal de Minas Gerais, um projeto realizado pelo governo do estado de Minas Gerais em parceria com o Instituto Estadual de Florestas (IEF) e a Universidade Federal de Lavras (UFLA) (SCOLFORO; OLIVEIRA; ACERBI JÚNIOR, 2008). Foram utilizados dados de 1325 árvores nativas cubadas no estado de Minas Gerais, com diâmetro maior que 5,0 cm, distribuídas em classes de diâmetros e altura, em diferentes bacias e sub-bacias hidrográficas e nas fisionomias florestais do cerradão ( 73 espécies), cerrado (118 espécies, inclui campo cerrado), decídua (22 espécies), ombrófila (71 espécies) e semidecídua (212 espécies), conforme Figura 1 . O número de árvores cubadas foi proporcional à densidade relativa das espécies obtidas no Inventário Florestal de Minas Gerais, critério esse aplicado às quatro espécies que apresentaram maior densidade relativa, e as demais foram agrupadas e computadas como se fossem uma única espécie. 


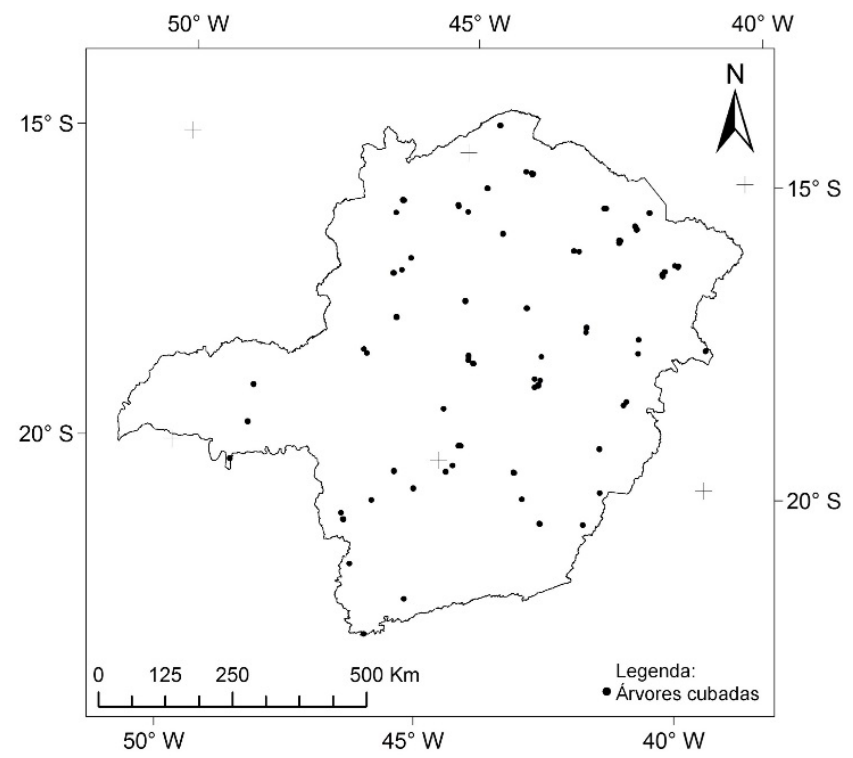

FIGURA 1: Locais em que foram amostradas as árvores, para cubagem, no estado de Minas Gerais. FIGURE 1: Places where trees were sampled for cubing, in Minas Gerais state, Brazil.

\section{Amostragem dos discos e determinação da densidade básica}

O procedimento de cubagem foi executado conforme o método de Huber. Após a cubagem, foram retirados cinco discos de madeira com espessura de $3,0 \mathrm{~cm}$ com casca, para manter a umidade, nas alturas correspondentes a 0, 25, 50,75 e 100\% da altura comercial das árvores (até o diâmetro de 3,0 cm), as quais foram codificadas neste estudo como A, B, C, D e E, respectivamente. Posteriormente, foram retiradas duas cunhas opostas em relação à medula de cada um dos cinco discos de madeira e removida a casca. A densidade básica de cada cunha foi obtida pela Equação 1, seguindo o procedimento descrito pela ABNT NBR

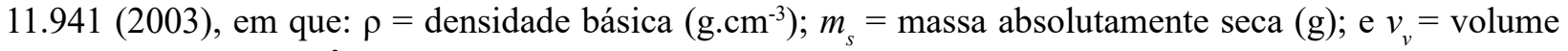
verde ou saturado $\left(\mathrm{cm}^{3}\right)$.

$$
\rho=m_{s} / v_{v}
$$

A densidade básica da madeira de cada disco foi obtida, a partir da média entre as duas cunhas, e a densidade básica geral do fuste da árvore foi calculada pela média aritmética entre as densidades dos cinco discos. Foi utilizada a média aritmética, em função dos estudos desenvolvidos por Vismara (2009), ao demonstrar que essa média estima melhor a densidade básica da árvore que a média ponderada, tendo como parâmetro a densidade obtida por gravimetria, a qual é considerada como mais precisa do que a técnica volumétrica por ser sujeita a menos fontes de erro.

\section{Análise dos dados}

Para verificar quais as melhores posições de amostragem e o número mais adequado de discos, para estimativa da densidade básica da madeira do fuste árvore, foram utilizados modelos de regressão linear. Na modelagem, a variável dependente foi definida como a "densidade básica média da madeira de cada fuste" $\left(\rho_{i}\right)$ e as variáveis independentes foram as "densidades básicas da madeira dos discos" $\left(\rho_{j n}\right)$ retirados das posições relativas $(j) \mathrm{A}, \mathrm{B}, \mathrm{C}, \mathrm{D}$ e E. Os modelos de regressão foram ajustados para todas as combinações entre os cinco discos tomados de $n$ a $n, \operatorname{com} n$ variando de um a quatro, em um total de 30 regressões (Tabela 1). Deve-se observar que não foi ajustada uma regressão, para a combinação dos cinco discos, uma vez que o valor de referência (parâmetro) foi dado pela média aritmética entre eles e, portanto, resultaria em uma equação com erro zero (modelo matemático). 
TABELA 1: Modelos de regressão linear ajustados a todas as combinações entre os cinco discos amostrados. TABLE 1: Linear regression models adjusted for each combination of the five sampled discs.

\begin{tabular}{clc}
\hline N. de Discos & \multicolumn{1}{c}{ Modelo de Regressão } & Combinações \\
\hline 1 & $\hat{\rho}_{i}=\beta_{0}+\beta_{1} \cdot \rho_{j_{1}}+\varepsilon$ & 5 \\
2 & $\hat{\rho}_{i}=\beta_{0}+\beta_{1} \cdot \rho_{j_{1}}+\beta_{2} \cdot \rho_{j_{2}}+\varepsilon$ & 10 \\
3 & $\hat{\rho}_{i}=\beta_{0}+\beta_{1} \cdot \rho_{j_{1}}+\beta_{2} \cdot \rho_{j_{2}}+\beta_{3} \cdot \rho_{j_{3}}+\varepsilon$ & 10 \\
4 & $\hat{\rho}_{i}=\beta_{0}+\beta_{1} \cdot \rho_{j_{1}}+\beta_{2} \cdot \rho_{j_{2}}+\beta_{3} \cdot \rho_{j_{3}}+\beta_{4} \cdot \rho_{j_{4}}+\varepsilon$ & 5 \\
5 & $\rho_{i}=\left(\rho_{A}+\rho_{B}+\rho_{C}+\rho_{D}+\rho_{E}\right) / 5$ (Parâmetro) & - \\
\hline
\end{tabular}

Em que: $\hat{\rho}_{i}=$ densidade básica estimada do fuste; $\beta_{n}=$ parâmetros a serem estimados; $\rho_{j_{n}}=$ densidade básica do disco da $j$-ésima posição (A, B, C, D ou E); $\rho_{i}=$ densidade básica do fuste (parâmetro); $\varepsilon=$ erro I.I.D.N. $\left(0, \sigma^{2}\right)$.

Os modelos de regressão foram ajustados pelo método dos mínimos quadrados ordinários (MQO), sob suposição de erros com distribuição normal, independentes e identicamente distribuídos com média zero e variância constante. Os modelos foram submetidos à análise gráfica dos resíduos padronizados e aos indicativos de precisão de ajuste Erro Padrão da Estimativa $\left(S_{y x}\right)$ e Critério de Informação de Akaike (AIC), representados nas Equações 2 e 3, respectivamente, em que: $n=$ número de observações; $p=$ número de parâmetros do modelo; $m v$ = máxima verossimilhança; demais termos já definidos. O critério de avaliação $A I C$ é um teste que permite uma comparação entre modelos não aninhados e penalizam aqueles com maior número de parâmetros, adequando-os ao conceito de parcimônia (WOLFINGER, 1993). Em ambos os indicadores, menores valores indicam melhor ajuste.

$S_{y x}=\sqrt{\frac{\sum_{i=1}^{n}\left(\rho_{i}-\hat{\rho}_{i}\right)^{2}}{n-p-1}}$

$A I C=-2 \ln (m v)+2 p$

Primeiramente, todos os procedimentos descritos e os ajustes dos modelos foram executados sem distinção da fitofisionomia, ou seja, para toda a base de dados, os quais foram tratados como "Equações Gerais", totalizando 30 equações, correspondentes a todas as combinações entre os discos. Depois, foi selecionada apenas uma equação por número de discos, totalizando quatro equações, as quais foram definidas como as melhores posições de amostragem, com base nos menores valores de $A I C$. Para definir o número mais adequado de discos, foi realizada comparação estatística entre essas quatro equações, também, em termos de $A I C$.

Em uma análise posterior, todos os procedimentos e os modelos foram ajustados, para cada fisionomia florestal separadamente, sendo elas o Cerradão, Cerrado (inclui Campo Cerrado), Decídua, Ombrófila e Semidecídua, as quais foram tratadas como "Equações por Fitofisionomia". Totalizaram 150 equações, das quais foram selecionadas 20 , quatro por fitofisionomia e cada uma das quatro correspondendo a uma combinação de um a quatro discos.

Prevendo que as Equações por Fitofisionomias pudessem apresentar coincidências, nas melhores posições de amostragem, quando comparadas às Equações Gerais, foi verificado se as equações coinciden- 
tes se diferiam estatisticamente, por meio da comparação entre os intervalos de confiança dos parâmetros $\left(\beta_{i}\right)$ em um nível de significância 0,05, de acordo com a Equação 4 (CHARNET et al., 1999), em que: $\hat{\beta}_{i}=$ estimativa do parâmetro; $E P$ = erro padrão associado a $\hat{\beta}_{i} ; t=$ valor crítico da distribuição $t$ de Student para $n-1$ graus de liberdade ao nível de significância $\alpha ; n=$ número total de observações.

$$
\hat{\beta}_{i} \pm E P_{\hat{\beta}_{i}} t_{(\alpha / 2 ; n-1)}
$$

Todas as análises e procedimentos foram executados com o auxílio do software R 2.9.12 (R DEVELOPMENT CORE TEAM, 2012).

\section{RESULTADOS E DISCUSSÃO}

\section{Equações Gerais}

Na Tabela 2 foram apresentadas as estatísticas do Critério de Informação de Akaike (AIC) para cada conjunto de discos para estimativa da densidade básica da madeira do fuste das árvores. Tem-se que o ponto ótimo de amostragem com apenas um disco foi representado pela posição $\mathrm{C}$ correspondente a $50 \%$ da altura da árvore, a qual apresentou o menor valor de $\operatorname{AIC}(-5092,9)$, seguida das posições $\mathrm{B}, \mathrm{D}, \mathrm{A}$ e E, demonstrando que as posições centrais são mais correlacionadas com a densidade geral da árvore, em relação a discos amostrados nas extremidades, quando apenas um disco do fuste é amostrado.

TABELA 2: Valor de AIC (Critério de Informação de Akaike) para as regressões referentes a cada combinação entre os discos amostrados.

TABLE 2: $\quad A I C$ value for the regressions for each combination between sampled discs.

\begin{tabular}{cccccccc}
\hline 1 Disco & $A I C$ & 2 Discos & $A I C$ & 3 Discos & $A I C$ & 4 Discos & AIC \\
\hline A & $-4453,7$ & AB & $-5535,6$ & ABC & $-6415,1$ & ABCD & $-7546,1$ \\
B & $-5020,0$ & AC & $-5939,0$ & ABD & $-6855,5$ & ABCE & $-8166,1$ \\
C & $-5092,9$ & AD & $-6017,1$ & ABE & $-6873,3$ & ABDE & $-8398,5$ \\
D & $-4608,1$ & AE & $-5612,8$ & ACD & $-6939,9$ & ACDE & $-8228,6$ \\
E & $-3868,5$ & BC & $-5909,8$ & ACE & $-7100,8$ & BCDE & $-7506,3$ \\
& & BD & $-6052,9$ & ADE & $-6789,2$ & & \\
& & BE & $-6013,0$ & BCD & $-6622,1$ & & \\
& & CD & $-5771,0$ & BCE & $-6901,5$ & & \\
& & CE & $-5767,3$ & BDE & $-6761,8$ & & \\
& & DE & $-5042,9$ & CDE & $-6227,6$ & & \\
\hline
\end{tabular}

Estudos semelhantes são mais comuns, para espécies do gênero Eucalyptus e, em boa parte destes trabalhos, mostra-se, geralmente, que apenas um disco garante estimativa adequada da densidade da árvore, sendo variável a posição da altura em que o disco deve ser retirado, porém, com maiores relatos de posições em torno do DAP ou $25 \%$ da altura comercial (FRANCO et al., 1998; BENJAMIN; BALLARIN, 2006; PÁDUA; TRUGILHO; LIMA, 2006). Alguns resultados com madeiras de espécies nativas sugerem, também, o disco próximo ao DAP (VALE; MARTINS; ARAÚJO, 1992) e até mesmo o disco próximo do topo (VISMARA, 2009), justamente, o qual apresentou a pior estimativa neste trabalho. Deve-se ressaltar que, em muitos trabalhos (FRANCO et al., 1998; PÁDUA; TRUGILHO; LIMA, 2006), utilizou-se como parâmetro a média ponderada da densidade e, assim as melhores posições de amostragem sempre tenderão a ser aquelas das posições com maior concentração de volume, ou seja, DAP e $25 \%$ da altura comercial. Ainda que alguns trabalhos mostrem diferenças não significativas entre a densidade média ponderada e a 
aritmética (NOGUEIRA; NELSON; FEARNSIDE, 2005; PÁDUA; TRUGILHO; LIMA, 2006; TRUGILHO, 2009), a posição de amostragem é totalmente influenciada.

Já para uma amostragem com dois discos, eles devem ser retirados nas posições BD correspondentes a 25 e $75 \%$ da altura da árvore, os quais apresentaram o menor valor de $A I C(-6052,9)$, seguidos das posições $\mathrm{AD}, \mathrm{BE}, \mathrm{AC}, \mathrm{BC}, \mathrm{CD}, \mathrm{CE}, \mathrm{AE}, \mathrm{AB}$ e $\mathrm{DE}$. Para uma amostra com três discos, a qual é muito comum na literatura, os pontos ótimos de amostragem correspondem às posições $\mathrm{ACE}$, que correspondem às posições de retiradas de 0,50 e $100 \%$ da altura da árvore, apresentando $A I C$ de $-7100,8$; seguidos das posições $\mathrm{ACD}, \mathrm{BCE}, \mathrm{ABE}, \mathrm{ABD}, \mathrm{ADE}, \mathrm{BDE}, \mathrm{BCD}, \mathrm{ABC}$ e $\mathrm{CDE}$. Por fim, para uma amostra com quatro discos, os pontos ótimos são representados por $\mathrm{ABDE}$ referentes a $0,25,75$ e $100 \%$ da altura da árvore, com AIC de -8398,5; seguidos das posições ACDE, ABCE, ABCD e BCDE.

Observa-se que as melhores posições de amostragem no fuste, quando analisado um maior número de discos, não mantêm relação com as melhores posições, quando analisada uma quantidade menor de discos. Nota-se que a melhor densidade geral da árvore apresenta tendência de distribuição mais simétrica e distribuída, com discos amostrados na base, meio e topo do fuste e não os discos mais correlacionados individualmente. Por exemplo, os discos A e E são individualmente os que se apresentaram inferiores, para estimativa da densidade geral da árvore, no entanto, eles aparecem nas melhores posições, quando são amostrados três e quatro discos, $\mathrm{ACE}$ e $\mathrm{ABDE}$, respectivamente.

$\mathrm{Na}$ Tabela 3 foram relacionados os quatro melhores ajustes acima, correspondentes a cada conjunto de discos para definição do número mais adequado para estimativa da densidade básica geral da madeira do fuste.

TABELA 3: Comparação dos valores de $A I C$ (Critério de Informação de Akaike) entre regressões com diferentes números de discos, correspondentes às melhores posições de amostragem.

TABLE 3: Comparison of $A I C$ value between regressions with different disc numbers, corresponding to the best sampling positions.

\begin{tabular}{cccccc}
\hline N. de Discos & Posições & $A I C$ & Teste & Diferença & P-valor \\
\hline 1 & C & $-5092,9$ & & & \\
2 & BD & $-6052,9$ & 1 vs 2 & 962,1 & 0 \\
3 & ACE & $-7100,8$ & 2 vs 3 & 1049,9 & 0 \\
4 & ABDE & $-8398,5$ & 3 vs 4 & 1299,7 & 0 \\
\hline
\end{tabular}

Claramente tem-se que a adição consecutiva de discos na amostra acarreta diferenças fortemente significativas ( $\mathrm{P}$-valor $<0,0001$ ), na estimativa da densidade básica da madeira do fuste, de modo que o uso de um menor número de discos, de maneira alguma, pode acarretar estimativas iguais ou mais precisas que uma amostra contendo mais discos. Resultados semelhantes podem ser vistos em Vismara (2009), ao afirmar que a menor discrepância da estimativa tende a ser menor quanto maior o número de discos combinados para determinação da densidade da madeira do tronco. De igual modo, Franco et al. (1998) e Benjamin e Ballarin (2006) afirmam que o maior número de discos garantiria estimativas mais eficientes. Possivelmente, o fato oposto só seria detectado caso houvesse uma amostragem com um número excessivo de discos amostrados como parâmetro.

Portanto, o uso de cinco discos sempre será o mais adequado, para se estimar a densidade básica geral da madeira da árvore, seguidos de quatro, três, dois e um disco. Conforme Benjamin e Ballarin (2006), quanto mais discos se amostram maior é a precisão. Contudo, essa melhora, muitas vezes, não é significativa para justificar um aumento muito grande no tamanho da amostra. Assim, deve-se considerar o erro associado ao uso de um número menor de discos, uma vez que a precisão mínima e os recursos disponíveis em estudos e pesquisas podem não exigir ou limitar o uso de um número maior de discos. Dessa maneira, na Tabela 4, são apresentadas as estimativas dos parâmetros e as estatísticas de ajuste das Equações Gerais, considerando um, dois, três e quatro discos para estimativa da densidade básica geral do fuste. 
TABELA 4: Equações Gerais e estatísticas de ajuste para estimativa da densidade do fuste a partir das melhores posições de amostragem.

TABLE 4: General Equations and fit statistics to estimate the stem density from the best sampling positions.

\begin{tabular}{|c|c|c|c|c|c|c|c|c|c|c|c|c|c|c|c|}
\hline \multirow{2}{*}{$\begin{array}{c}\text { N. } \\
\text { Discos }\end{array}$} & \multicolumn{13}{|c|}{$\begin{array}{l}\text { Modelo/ } \\
\text { Equação }\end{array}$} & \multirow{2}{*}{$\begin{array}{c}\mathrm{S}_{\mathrm{yx}} \\
\left(\mathrm{g} \cdot \mathrm{cm}^{-3}\right)\end{array}$} & \multirow{2}{*}{$\begin{array}{l}S_{y x} \\
(\%)\end{array}$} \\
\hline & $\beta_{0}$ & + & $\beta_{1}$ & $\rho_{j 1}$ & + & $\beta_{2}$ & $\rho_{i 2}$ & + & $\beta_{3}$ & $\rho_{i 3}$ & + & $\beta_{4}$ & $\rho_{i 4}$ & & \\
\hline 1 & 0,061 & + & 0,896 & $\mathrm{C}$ & & & & & & & & & & 0,03 & 5,16 \\
\hline 2 & 0,031 & + & 0,507 & B & + & 0,443 & $\mathrm{D}$ & & & & & & & 0,021 & 3,48 \\
\hline 3 & 0,008 & + & 0,292 & A & + & 0,411 & $\mathrm{C}$ & + & 0,279 & E & & & & 0,013 & 2,27 \\
\hline 4 & $0,0019^{\text {ns }}$ & + & 0,229 & A & + & 0,279 & $\mathrm{~B}$ & + & 0,267 & $\mathrm{D}$ & + & 0,222 & E & 0,008 & 1,33 \\
\hline
\end{tabular}

Em que: ns = parâmetro não significativo a 0,05 de significância, demais parâmetros sem "ns" são significativos a 0,0001 .

Independentemente do número de discos considerados, todas as equações apresentaram parâmetros altamente significativos (P-valor $<0,0001$ ), exceto pelo intercepto $\left(\beta_{0}\right)$ da equação com quatro discos. Entretanto, mesmo não significativo, esse parâmetro foi mantido, o que significa que, em termos práticos, a regressão passará pela origem. De acordo com Gujarati e Porter (2011), a menos que exista uma expectativa a priori muito forte quanto ao uso de um modelo de regressão sem intercepto, é aconselhável que o parâmetro esteja presente. Dessa maneira, evitam-se problemas com possíveis erros de especificação do modelo, dado que, para os demais casos, $\beta_{0}$ foi significativo.

As diferenças da Tabela 4 correspondem a uma média relativa de, aproximadamente, $35 \%$ de redução no $S_{y x}$ quando se adiciona um disco, fato que justifica a diferença fortemente significativa entre eles. Entretanto, os valores absolutos percentuais podem ser considerados baixos, mesmo para a estimativa com apenas um disco. $\mathrm{Na}$ área florestal, dificilmente se têm equações ajustadas com erro em torno de $5 \%$, independentemente das variáveis. Isso implica a possibilidade de estimar a densidade básica da madeira do fuste com pelo menos um disco amostrado, na posição já indicada. Obviamente que, como o acréscimo de um disco acarreta redução substancial no erro, o uso de apenas um disco deve ser considerado em circunstâncias limitantes em que não é possível a amostragem de mais discos, ou ainda, quando há perda das informações coletadas por alguma eventualidade. Logo, foram apresentadas quatro possibilidades, para estimar a densidade geral do fuste, a partir de um número menor de discos amostrados, os quais garantem alta precisão e permitem ao pesquisador adequar uma equação à sua realidade de pesquisa.

Deve-se atentar para a importância de se utilizar as equações propostas para estimativa e não os valores absolutos ou valores médios de densidade da madeira dos discos retirados nas posições indicadas. Percebe-se, por meio das equações ajustadas, que os discos não apresentam "pesos" iguais, por exemplo, caso fossem amostrados discos nas posições ACE, uma possível média entres eles, para estimativa da densidade do fuste, implicaria peso de 0,333 para cada um. No entanto observa-se que eles têm pesos respectivos de 0,$2922 ; 0,4108$ e 0,2794 , dados pelos parâmetros da regressão, além de uma correção de 0,008 g.cm ${ }^{-3}$ pelo intercepto. Assim, o disco da posição C tem peso maior que os discos de A e E, nessa ordem. De maneira análoga para as outras equações, observa-se que os discos centrais $\mathrm{B}, \mathrm{C}$ e D sempre tendem a ter maior peso que os discos externos A e E. Portanto as equações propostas, além de indicar as melhores posições de amostragem, apresentam uma espécie de "correção" dos valores absolutos de densidade dos discos para garantir erro mínimo na estimativa da densidade geral da madeira do fuste.

Basta, afinal, verificar se a distribuição gráfica de resíduos atende aos pressupostos estabelecidos pela regressão (Figura 2). Destacam-se resíduos bem distribuídos, sem tendência, com concentração das observações em torno de dois desvios padrões, sem indícios de heterocedasticidade, independentemente do número de discos. Os gráficos se diferenciam apenas quanto à concentração de observações em torno de zero. Nota-se que, à medida que se adicionam discos ao modelo, a nuvem de pontos tende a se agrupar em torno do eixo, novamente comprovando as estimativas altamente significativas entre eles. De acordo o histograma auxiliar lateral, os resíduos não apresentaram problemas de normalidade. 

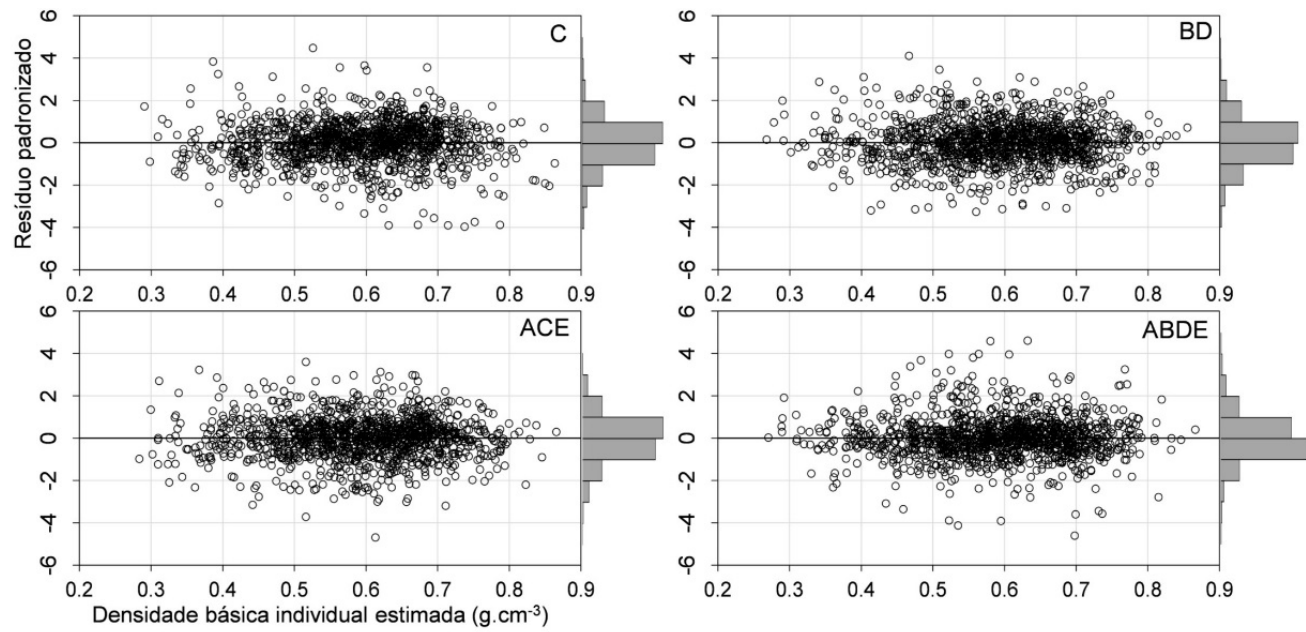

FIGURA 2: Gráficos de resíduos padronizados das Equações Gerais referentes aos discos e posições C, BD, ACE e ABDE.

FIGURE 2: Standardized residuals plots of General Equations in relation to discs and positions C, BD, ACE and ABDE.

Por isso, após todas as estatísticas de ajuste e análise de resíduos, pode-se afirmar que as Equações Gerais são precisas e não apresentam problemas quanto às pressuposições estabelecidas sobre os resíduos, garantindo estimativas adequadas da densidade básica da madeira do fuste com um número menor de dis$\cos$.

\section{Equações por Fitofisionomia}

As melhores posições de amostragem por fitofisionomias já pré-selecionadas, em função do Critério de Informação de Akaike $(A I C)$, são apresentadas na Tabela 5, sendo necessário destacar que, eventualmente, algumas combinações, para um mesmo número de discos, foram estatisticamente idênticas quanto $A I C$ e, em consequência, a melhor posição apresentada foi aquela com melhor distribuição de resíduos.

Em uma visão geral, as fitofisionomias apresentam comportamento diferenciado quanto à melhor posição de amostragem dos discos, apenas as formações de Cerradão e Cerrado foram idênticas às Equações Gerais para todos os números de discos. A fisionomia Decídua apresentou as mesmas posições que a Equação Geral para um (C) e quatro discos (ABDE), as posições para dois e três foram $\mathrm{CD}$ e $\mathrm{ACD}$, respectivamente. No caso da Ombrófila, apenas as posições com quatro discos coincidiram com a Equação Geral (ABDE) e, já para um, dois e três discos, foram B, BC e ABE, respectivamente. No caso das florestas Semidecíduas, nenhuma combinação de discos foi igual às Equações Gerais, sendo as melhores posições para um, dois, três e quatro discos, representadas por $\mathrm{B}, \mathrm{AD}, \mathrm{ACD}$ e ACDE, respectivamente.

TABELA 5: Melhores posições de amostragem de discos para estimar a densidade do fuste em cada fitofisionomia. TABLE 5: Best sampling positions of discs for estimate the stem density in each phytophysiognomy.

\begin{tabular}{ccccccc}
\hline N. de & \multicolumn{5}{c}{ Fitofisionomia } \\
\cline { 2 - 7 } discos & Geral & Cerradão & Cerrado & Decídua & Ombrófila & Semidecídua \\
\hline 1 & $\mathrm{C}$ & $\mathrm{C}$ & $\mathrm{C}$ & $\mathrm{C}$ & $\mathrm{B}$ & $\mathrm{B}$ \\
2 & $\mathrm{BD}$ & $\mathrm{BD}$ & $\mathrm{BD}$ & $\mathrm{CD}$ & $\mathrm{BC}$ & $\mathrm{AD}$ \\
3 & $\mathrm{ACE}$ & $\mathrm{ACE}$ & $\mathrm{ACE}$ & $\mathrm{ACD}$ & $\mathrm{ABE}$ & $\mathrm{ACD}$ \\
4 & $\mathrm{ABDE}$ & $\mathrm{ABDE}$ & $\mathrm{ABDE}$ & $\mathrm{ABDE}$ & $\mathrm{ABDE}$ & $\mathrm{ACDE}$ \\
\hline
\end{tabular}


O simples fato de se conhecer a formação florestal estudada pode auxiliar ainda mais na estimativa da densidade básica da madeira do fuste, uma vez que as melhores posições de amostragem podem variar, em função dela mesma, assim, o uso dos discos mais adequados pode garantir o menor erro da estimativa.

$\mathrm{Na}$ Tabela 6 são apresentadas as estimativas dos parâmetros e as estatísticas de ajuste das Equações por Fitofisionomia.

TABELA 6: Equações e estatísticas de ajuste para estimativa da densidade do fuste por fitofisionomia, a partir das melhores posições de amostragem.

TABLE 6: Equations and fit statistics to estimate the stem density by phytophysiognomy from the best sampling positions.

\begin{tabular}{|c|c|c|c|c|c|c|c|c|c|c|c|c|c|c|c|c|}
\hline \multirow[t]{2}{*}{ Fitofisionomia } & \multirow[t]{2}{*}{ ND } & \multicolumn{13}{|c|}{$\begin{array}{l}\text { Modelo/ } \\
\text { Equação }\end{array}$} & \multirow{2}{*}{$\begin{array}{c}\mathrm{S}_{\mathrm{yx}} \\
\left(\mathrm{g} . \mathrm{cm}^{-3}\right)\end{array}$} & \multirow{2}{*}{$\begin{array}{l}\mathrm{S}_{\mathrm{yx}} \\
(\%)\end{array}$} \\
\hline & & $\beta_{0}$ & + & $\beta_{1}$ & $\rho_{j 1}$ & + & $\beta_{2}$ & $\rho_{j 2}$ & + & $\beta_{3}$ & $\rho_{j 3}$ & + & $\beta_{4}$ & $\rho_{j 4}$ & & \\
\hline \multirow[t]{4}{*}{ Cerradão } & 1 & 0,104 & + & 0,808 & $\mathrm{C}$ & & & & & & & & & & 0,029 & 5,1 \\
\hline & $2^{*}$ & 0,032 & + & 0,499 & B & + & 0,458 & $\mathrm{D}$ & & & & & & & 0,021 & 3,71 \\
\hline & $3^{*}$ & 0,019 & + & 0,276 & $\mathrm{~A}$ & + & 0,424 & $\mathrm{C}$ & + & 0,256 & $\mathrm{E}$ & & & & 0,013 & 2,24 \\
\hline & $4^{*}$ & $-0,0074^{\mathrm{ns}}$ & + & 0,224 & A & + & 0,308 & B & + & 0,274 & $\mathrm{D}$ & + & 0,211 & $\mathrm{E}$ & 0,008 & 1,43 \\
\hline \multirow[t]{4}{*}{ Cerrado } & $1^{*}$ & 0,077 & + & 0,872 & $\mathrm{C}$ & & & & & & & & & & 0,028 & 4,59 \\
\hline & $2^{*}$ & 0,039 & + & 0,485 & B & + & 0,452 & D & & & & & & & 0,019 & 3,12 \\
\hline & $3^{*}$ & 0,008 & + & 0,305 & A & + & 0,410 & $\mathrm{C}$ & + & 0,267 & $\mathrm{E}$ & & & & 0,013 & 2,05 \\
\hline & $4^{*}$ & $0,0008^{\mathrm{ns}}$ & + & 0,232 & A & + & 0,274 & B & + & 0,272 & D & + & 0,219 & $\mathrm{E}$ & 0,007 & 1,21 \\
\hline \multirow[t]{4}{*}{ Decídua } & $1^{*}$ & 0,062 & + & 0,924 & $\mathrm{C}$ & & & & & & & & & & 0,021 & 3,1 \\
\hline & 2 & 0,035 & + & 0,668 & $\mathrm{C}$ & + & 0,304 & $\mathrm{D}$ & & & & & & & 0,016 & 2,36 \\
\hline & 3 & $0,0143^{\text {ns }}$ & + & 0,211 & A & + & 0,432 & $\mathrm{C}$ & + & 0,334 & $\mathrm{D}$ & & & & 0,01 & 1,46 \\
\hline & $4^{*}$ & $-0,0041^{\mathrm{ns}}$ & + & 0,232 & A & + & 0,298 & B & + & 0,240 & $\mathrm{D}$ & + & 0,232 & $\mathrm{E}$ & 0,005 & 0,75 \\
\hline \multirow[t]{4}{*}{ Ombrófila } & 1 & 0,096 & + & 0,804 & $\mathrm{~B}$ & & & & & & & & & & 0,031 & 6,33 \\
\hline & 2 & 0,040 & + & 0,440 & B & + & 0,477 & $\mathrm{C}$ & & & & & & & 0,021 & 4,32 \\
\hline & 3 & 0,024 & + & 0,258 & A & + & 0,35 & B & + & 0,342 & E & & & & 0,015 & 3,09 \\
\hline & 4 & $0,0073^{\text {ns }}$ & + & 0,238 & A & + & 0,26 & B & + & 0,219 & $\mathrm{D}$ & + & 0,27 & $\mathrm{E}$ & 0,008 & 1,64 \\
\hline \multirow[t]{4}{*}{ Semidecídua } & 1 & 0,072 & + & 0,859 & $\mathrm{~B}$ & & & & & & & & & & 0,031 & 5,55 \\
\hline & 2 & 0,008 & + & 0,404 & A & + & 0,563 & $\mathrm{D}$ & & & & & & & 0,02 & 3,5 \\
\hline & 3 & $0,0050^{\text {ns }}$ & + & 0,304 & A & + & 0,306 & $\mathrm{C}$ & + & 0,366 & $\mathrm{D}$ & & & & 0,013 & 2,39 \\
\hline & 4 & $-0,0027^{\mathrm{ns}}$ & + & 0,275 & A & + & 0,270 & $\mathrm{C}$ & + & 0,254 & $\mathrm{D}$ & + & 0,205 & E & 0,008 & 1,48 \\
\hline
\end{tabular}

Em que: $n s$ = parâmetro não significativo a 0,05 de significância, demais parâmetros sem "ns" são fortemente significativos a 0,$0001 ; *$ * equação estatisticamente idêntica à Equação Geral, em relação ao respectivo número de discos; $\mathrm{ND}=$ número de discos.

Quanto à significância dos parâmetros $\beta_{1}, \beta_{2}, \beta_{3}$ e $\beta_{4}$, associados aos discos das equações apresentadas, todos foram altamente significativos (P-valor $<0,0001$ ), ao contrário de diversos interceptos $\left(\beta_{0}\right)$ que, entretanto, foram mantidos por questões já relatadas. Os erros padrão das estimativas $\left(S_{y x}\right)$ apresentaram praticamente as mesmas proporções estabelecidas nas Equações Gerais, para cada número de discos, com exceção da fitofisionomia Ombrófila que apresentou cerca de um ponto percentual de aumento no erro para as estimativas com dois, três e quatro discos. Nota-se, também, que, dentre as 20 equações, apenas nove 
delas são estatisticamente idênticas às Equações Gerais correspondentes e, dessa maneira, as 11 demais devem ser priorizadas em detrimento das Equações Gerais, quando há identificação da fitofisionomia estudada, uma vez que irão garantir estimativas mais precisas da densidade geral do fuste.

A mesma analogia feita para as Equações Gerais, quanto aos "pesos" de cada disco, pode ser observada por fitofisionomia. Geralmente, os discos centrais tendem a ser mais importantes, para as estimativas em relação aos discos externos, contudo, há ocorrência de duas exceções: na equação com quatro discos (ACDE) da florestal Semidecídua, em que o disco A apresenta peso maior $(0,2753)$ que C, D e E; e na Ombrófila, na equação com quatro discos (ABDE), na qual o disco do topo E obteve peso maior que os demais.

Por fim, a análise gráfica de resíduos de cada modelo ajustado por fitofisionomia e por número de discos é apresentada na Figura 3, excluídas aquelas nove que se apresentaram estatisticamente idênticas às Equações Gerais correspondentes.

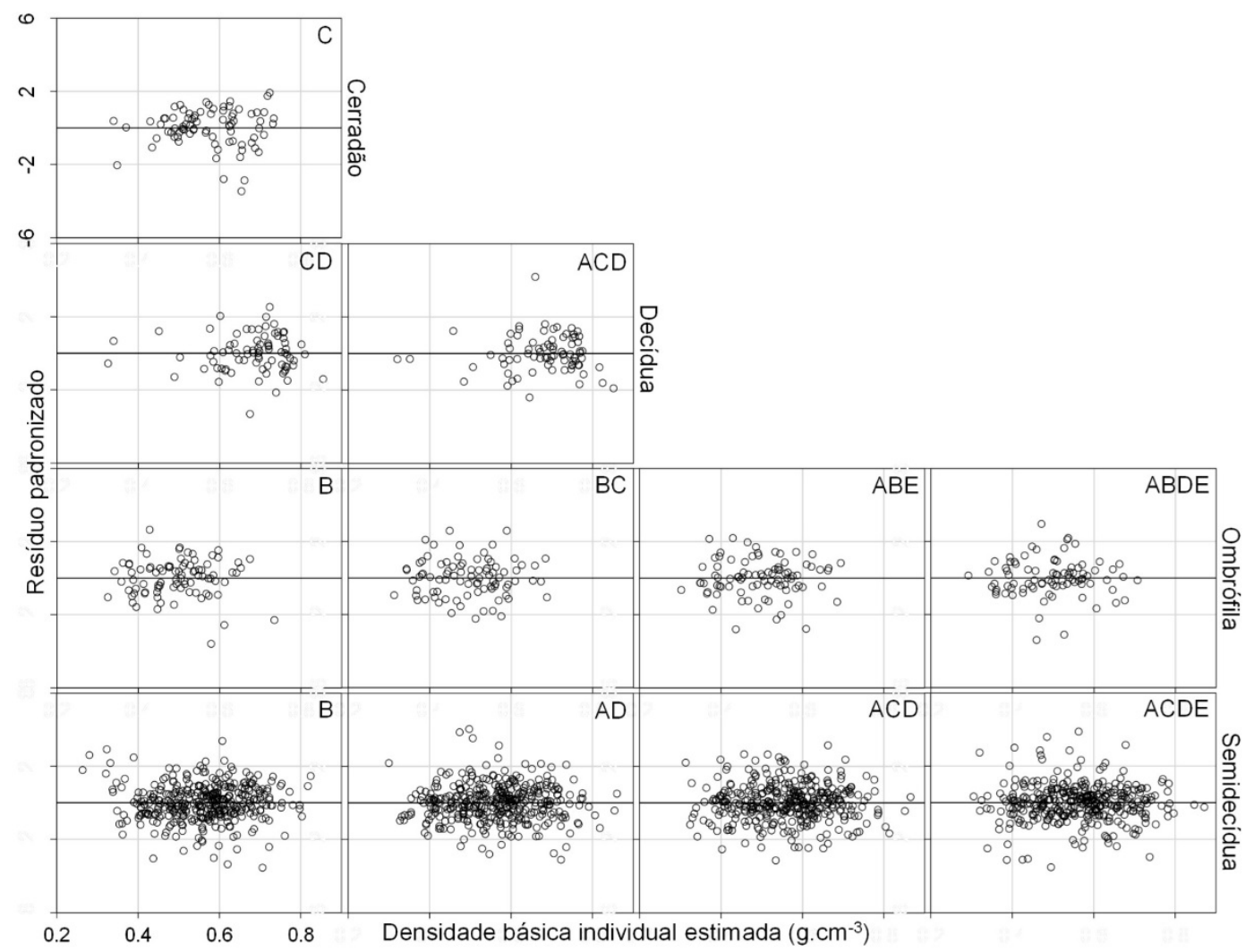

FIGURA 3: Gráficos de resíduos padronizados das Equações por Fitofisionomia para as combinações de discos estatisticamente distintas das Equações Gerais.

FIGURE 3: Standardized residuals plots of Equations by Phytophysiognomy, for the disc combinations statistically distinct of the General Equations.

Em geral, percebe-se um padrão de simetria, com observações distribuídas aleatoriamente, sem tendência, com concentração em torno de dois desvios, sem indícios de dependência ou heterocedasticidade, independentemente da fitofisionomia ou número de discos.

As divergências quanto às posições ótimas dos discos, bem como a densidade básica da madeira do fuste das árvores das fitofisionomias entre si, podem estar associadas à composição de espécies e a fatores ambientais condicionantes dessas fitofisionomias. Esses fatores influenciam a variabilidade da densidade da madeira uma vez que afetam a taxa de crescimento das espécies florestais (RIGATTO; DEDECEK; MATOS, 2004). O efeito no crescimento é influenciado pelos processos fisiológicos das árvores. Como resposta, as árvores adaptam seus processos fisiológicos, de acordo com condições ambientais, ao reflexo na atividade cambial e à anatomia do lenho (VILLAR et al., 1997). Como a densidade da madeira é resultado 
da combinação da quantidade e da distribuição das diferentes células lenhosas, bem como pela quantidade de extrativos (ROQUE; TOMAZELLO FILHO, 2009), as variações edafoclimáticas certamente irão afetá-la de alguma maneira. Como a ocorrência das diferentes fitofisionomias está relacionada a diferentes fatores edáficos, topográficos e climáticos (RODRIGUES et al., 2003; AGNES et al., 2007), espera-se que os mesmos fatores que condicionam as fitofisionomias, também, reflitam efeitos sobre a densidade da sua madeira (OLIVEIRA et al., 2012), nesse caso, possivelmente influenciando a densidade da madeira dentro do fuste, no sentido base-topo e, consequentemente, alterando as posições de amostragem mais correlacionadas com toda a árvore.

\section{CONCLUSÕES}

De maneira geral, a melhor posição de amostragem para estimativa da densidade básica da madeira do fuste com apenas um disco deve ser efetuada na metade do fuste principal. Utilizando-se dois discos, a estimativa da densidade pode ser obtida com precisão, se esses discos forem amostrados nas posições $25 \%$ e $75 \%$ da altura. Já com três discos, as posições devem ser 0, 50 e $100 \%$ da altura. Essas posições variam, significativamente, de acordo com a fitofisionomia estudada. A estimativa mais precisa da densidade básica do fuste sempre será dada por aquela que apresentar o maior número de discos amostrados, entretanto, é possível estimar, com alta precisão, a densidade básica da madeira do fuste com um número menor de discos, podendo ser utilizado até um único disco, desde que amostrado em posições específicas do fuste e utilizando-se as equações propostas neste trabalho, de acordo com a fitofisionomia estudada.

Por fim, espera-se que o presente trabalho possa contribuir com demais estudos de biomassa e de carbono em florestas nativas, sobretudo, com a redução do esforço amostral, sem que haja perda em precisão e, consequentemente, com a economia de tempo e recursos.

\section{REFERÊNCIAS}

AGNES, C. C. et al. Fatores ecológicos condicionantes da vegetação do cerradão. Caderno de Pesquisa: Série Biologia, Santa Cruz do Sul, v. 19, n. 3, p. 25-37, 2007.

ASSOCIAÇÃO BRASILEIRA DE NORMAS TÉCNICAS. NBR 11941: Madeira - Determinação da densidade básica. Rio de Janeiro, 2003.

BENJAMIN. C. A.; BALLARIN, A. W. Avaliação da precisão de amostras retiradas ao longo do fuste das árvores na determinação da densidade básica da madeira de populações florestais. In: ENCONTRO BRASILEIRO EM MADEIRAS E EM ESTRUTURAS DE MADEIRAS, 10., 2006, São Pedro. Anais... São Pedro: Unesp, 2006. p. 1-15.

CHARNET, R. et al. Análise de modelos de regressão linear com aplicações. São Paulo: Unicamp, 1999. $354 p$.

FEARNSIDE, P. M. Quantificação do serviço ambiental do carbono nas florestas amazônicas brasileiras. Oecologia Brasiliensis, Rio de Janeiro, v. 12, n. 4, p. 743-756, 2008.

FRANCO, E. J. et al. Eficiência na estimativa do peso seco para árvores individuais e definição do ponto ótimo de amostragem para determinação da densidade básica de Eucalyptus camaldulensis. Ciência Florestal, Santa Maria, v. 8, n. 1, p. 77-92. 1998.

GUJARATI, D.; PORTER, D. C. Econometria básica. 5. ed. Porto Alegre: AMGH, 2011. 924 p.

NOGUEIRA, E. M. et al. Wood density in forests of Brazil's 'arc of deforestation': Implications for biomass and flux of carbon from land-use change in Amazonia. Forest Ecology and Management, Amsterdam, v. 248, p. 119-135, 2007.

NOGUEIRA, E. M.; NELSON, B. W.; FEARNSIDE, P. M. Wood density in dense forest in central Amazonia, Brazil. Forest Ecology and Management, Amsterdam, v. 208, p. 261-286, 2005.

OLIVEIRA, G. M. V. et al. Efeito do Ambiente sobre a Densidade da Madeira nas Fitofisionomias no Estado de Minas Gerais. Cerne, Lavras, v. 18, n. 2, p. 345-352, abr./jun. 2012.

PÁDUA, F. A.; TRUGILHO, P. F.; LIMA, J. T. Amostragem da madeira para avaliação da densidade básica em Eucalyptus. In: ENCONTRO BRASILEIRO EM MADEIRAS E EM ESTRUTURAS DE 
MADEIRAS, 10., 2006, São Pedro. Anais... São Pedro: Unesp, 2006. p. 1-9.

R DEVELOPMENT CORE TEAM. R: a language and environment for statistical computing. Vienna: R Foundation for Statistical Computing, 2012.

RIGATTO, P. A.; DEDECEK, R. A.; MATOS, J. L. M. Influência dos atributos do solo sobre a qualidade da madeira de pinus taeda para produção de celulose kraft. Revista Árvore, Viçosa, MG, v. 28, n. 2, p. 267-273, 2004.

RODRIGUES, L. A. et al. Florística e estrutura da comunidade arbórea de um fragmento florestal em Luminárias, MG. Acta Botanica Brasilica, Feira de Santana, v. 17, p. 71-97, 2003.

ROQUE, R. M.; TOMAZELLO FILHO, M. Variação radial da estrutura anatômica do lenho de árvores de Gmelina arborea em diferentes condições de clima e de manejo na Costa Rica. Scientia Forestalis, Piracicaba, v. 37, n. 83, p. 273-285, set. 2009.

SANQUETTA, C. R. Métodos de determinação de biomassa florestal. In: SANQUETTA, C. R. et al. (Ed.). As florestas e o carbono. [s. 1.: s. n.], 2002. p. 119-140.

SCOLFORO, J. R. S. et al. Estimativas de volume, peso seco, peso de óleo e quantidade de moirões para a candeia (Eremanthus erythropappus (DC.) MacLeish). Cerne, Lavras, v. 10 n. 1, p. 87-102, jan./jun. 2004. SCOLFORO, J. R. S.; OLIVEIRAA. D.; ACERBI JÚNIOR, F. W. Inventário florestal de Minas Gerais: equações de volume, peso de matéria seca e carbono para diferentes fisionomias da flora nativa. Lavras: Editora UFLA, 2008. p. 216.

SILVEIRA, P. Estimativa da biomassa e carbono acima do solo em um fragmento de floresta ombrófila densa utilizando o método da derivação do volume comercial. Floresta, Curitiba, v. 40, n. 4, p. 789-800, out./dez. 2010.

SILVEIRA, P. et al. O estado da arte na estimativa de biomassa e carbono em formações florestais. Floresta, Curitiba, v. 38, n. 1, jan./mar. 2008.

TRUGILHO, P. F. Densidade básica e estimativa de massa seca e de lignina na madeira em espécies de Eucalyptus. Ciência e agrotecnologia, Lavras, v. 33, n. 5, p. 1228-1239, set./out. 2009.

VALE, T.; MARTINS, I. S.; ARAÚJO, W. L. O. Estudo da densidade de três espécies do cerrado. Revista Árvore, Viçosa, MG, v. 16, n. 2, p. 209-217, maio/ago. 1992.

VIEIRA, G.; SANQUETTA, C. R.; BARBEIRO, L. S. S. Estoque individual de biomassa e carbono em Nectandra grandiflora Nees (canela-amarela). Floresta, Curitiba, v. 39, n. 3, p. 547-554, jul./set. 2009.

VILLAR, P. et al. Stem xylem features in three Quercus (Fagaceae) species along a climatic gradient in NE Spain. Trees Structure and Function, [s. 1.], v. 12, n. 2, p. 90-96, 1997.

VISMARA, E. S. Mensuração da biomassa e construção de modelos para construção de equações de biomassa. 2009. 102 f. Dissertação (Mestrado) - Escola Superior de Agricultura Luiz de Queiroz, Piracicaba, 2009.

WOLFINGER, R. Covariance structure selection in general mixed models. Communications in Statistics - Simulation, Ontario, v. 22, n. 4, p. 1079-1106, 1993.

WOODCOCK, D.W. Wood specific gravity of trees and forest types in the Southern Peruvian Amazon. Acta Amazonica, Manaus, v. 30, n. 4, p. 589-599, 2000. 